УДК 517.4

D. Salim, W. S. Budhi, Y. Soeharyadi

\title{
ON ROUGH MAXIMAL INEQUALITIES: AN EXTENSION OF FEFFERMAN-STEIN RESULTS
}

D. Salim, W. S. Budhi, Y. Soeharyadi. On rough maximal inequalities: an extension of Fefferman-Stein results, Mat. Stud. 52 (2019), 185-194.

We prove some vector-valued inequalities for a rough maximal operator on Lebesgue spaces. These results are an extension of Fefferman-Stein (1971) and Sawano (2006) since the rough maximal operator is a generalization of the Hardy-Littlewood maximal operator and also a fractional maximal operator, respectively. We also establish some vector-valued inequalities for a rough maximal operator on Morrey spaces.

1. Introduction. Let $f$ be a measurable function on $\mathbb{R}^{n}$. Let also $\Omega$ be a homogeneous function of degree zero on $\mathbb{R}^{n}$. For $\alpha \in[0, n)$, the rough maximal operator $M_{\Omega, \alpha}$ maps the function $f$ to the rough maximal function $M_{\Omega, \alpha} f$ which is given by

$$
M_{\Omega, \alpha} f(x):=\sup _{r>0} r^{\alpha-n} \int_{B(x, r)}|\Omega(x-y)||f(y)| d y, \quad x \in \mathbb{R}^{n}
$$

where $B(x, r)$ denotes the open ball centered in $x$ with radius $r>0$. For $\alpha \in(0, n)$, the operator $M_{1, \alpha}$ is known as a fractional maximal operator. The operator $M_{1,0}$ is well known as the Hardy-Littlewood maximal operator.

We write $\mathbf{f}$ to denote a sequence of measurable functions $\left\{f_{j}\right\}_{j=1}^{\infty}$ on $\mathbb{R}^{n}$. We also write $M_{\Omega, \alpha} \mathbf{f}$ to denote the rough maximal function sequence $\left\{M_{\Omega, \alpha} f_{j}\right\}_{j=1}^{\infty}$. We use $a \lesssim b$ to indicate that there exists $c>0$ such that $a \leq c b$. We express Fefferman-Stein maximal inequalities using this notations as follows.

Proposition 1 ([5], Theorem 1). Let $u \in(1, \infty)$. Then, for $p \in(1, \infty)$

$$
\left\|M_{1,0} \mathbf{f}\right\|_{L^{p}\left(\ell^{u}\right)}:=\|\| M_{1,0} \mathbf{f}(\cdot)\left\|_{\ell^{u}}\right\|_{L^{p}}=\left(\int_{\mathbb{R}^{n}}\left(\sum_{j=1}^{\infty} M_{1,0} f_{j}(x)\right)^{\frac{p}{u}} d x\right)^{\frac{1}{p}} \lesssim\|\mathbf{f}\|_{L^{p}\left(\ell^{u}\right)},
$$

and

$$
\left\|M_{1,0} \mathbf{f}\right\|_{W L^{1}\left(\ell^{u}\right)}:=\|\| M_{1,0} \mathbf{f}(\cdot)\left\|_{\ell^{u}}\right\|_{W L^{1}}=\sup _{\tau>0} \tau\left(\int_{\left\{x \in \mathbb{R}^{n} ;\left\|M_{1,0} \mathbf{f}(x)\right\|_{\left.\ell^{u}>\tau\right\}}\right.} d x\right) \lesssim\|\mathbf{f}\|_{L^{1}\left(\ell^{u}\right)} .
$$

2010 Mathematics Subject Classification: 42B25, $42 \mathrm{~B} 35$.

Keywords: rough maximal operator; vector-valued inequality; Fefferman-Stein maximal inequality; Morrey space.

doi:10.30970/ms.52.2.185-194

(C) D. Salim, W. S. Budhi, Y. Soeharyadi, 2019 
For any $j \in \mathbb{N}$, we have

$$
\left|f_{j}(x)\right| \leq \sup _{j \in \mathbb{N}}\left|f_{j}(x)\right|=\|\mathbf{f}(x)\|_{\ell^{\infty}}
$$

for almost every $x \in \mathbb{R}^{n}$. Therefore,

$$
\left\|M_{1,0} \mathbf{f}(x)\right\|_{\ell^{\infty}} \leq\left\|M_{1,0}\right\| \mathbf{f}(\cdot)\left\|_{\ell^{\infty}}(x)\right\|_{\ell^{\infty}}=M_{1,0}\left(\|\mathbf{f}(\cdot)\|_{\ell^{\infty}}\right)(x) .
$$

Hence, inequality (1) and (2) hold for $u=\infty$ due to inequality (3) and the boundedness of $M_{1,0}$ on Lebesgue spaces. However, inequality (1) and (2) do not hold for $u=1$ (see [13]).

In 2006, Sawano proved the following fractional maximal inequalities.

Proposition 2 ([12], Theorem 2). Let $u \in[1, \infty], \alpha \in(0, n)$, and $\frac{1}{q}=\frac{1}{p}-\frac{\alpha}{n}$. Then for $p \in\left(1, \frac{n}{\alpha}\right)$,

$$
\left\|M_{1, \alpha} \mathbf{f}\right\|_{L^{q}\left(\ell^{u}\right)} \lesssim\|\mathbf{f}\|_{L^{p}\left(\ell^{u}\right)}, \quad\left\|M_{1, \alpha} \mathbf{f}\right\|_{W L^{\frac{n}{n-\alpha}\left(\ell^{u}\right)}} \lesssim\|\mathbf{f}\|_{L^{1}\left(\ell^{u}\right)} .
$$

Our main aim is to prove the vector-valued inequality for the rough maximal operator $M_{\Omega, \alpha}$ as an extension of Proposition 1 and Proposition 2. We use $a \lesssim_{\Omega} b$ to express that there exists $c>0$ which depends on $\Omega$ such that $a \leq c b$. In Section 2, we prove the following inequalities.

$$
\left\|M_{\Omega, \alpha} \mathbf{f}\right\|_{L^{q}\left(\ell^{u}\right)} \lesssim \Omega\|\mathbf{f}\|_{L^{p}\left(\ell^{u}\right)},
$$

and

$$
\left\|M_{\Omega, \alpha} \mathbf{f}\right\|_{W L^{\frac{n}{n-\alpha}\left(\ell^{u}\right)}} \lesssim \Omega\|\mathbf{f}\|_{L^{1}\left(\ell^{u}\right)} .
$$

Note that if $\mathbf{f}=\left\{f_{j}\right\}_{j=1}^{\infty}$ with $f_{1}=f, f_{j}=0$ for $j>2$, then both inequalities (4) and (5) mean the boundedness of $M_{\Omega, \alpha}$ in Lebesgue spaces (see [10, Theorem 2] and [6, Section 5.2.3]). For such $\mathbf{f}$, the Calderón-Zygmund decomposition and the covering property are main keys to prove inequality (5), which are applied to $\|\mathbf{f}\|_{\ell^{u}}=|f| \in L^{1}$. In more general cases of $\mathbf{f}$, although the covering property applies directly to $\|\mathbf{f}\|_{\ell^{u}} \in L^{1}$, the CalderónZygmund decomposition does not apply directly to $\|\mathbf{f}\|_{\ell^{u}}$. To obtain the desired result, we apply Calderón-Zygmund decomposition to each $f_{j} \in \mathbf{f}$ instead (see Theorem 3 ).

Let $S^{n-1}=\left\{x \in \mathbb{R}^{n} ;|x|=1\right\}$. If $\Omega \in L^{\infty}\left(S^{n-1}\right)$, then $M_{\Omega, \alpha} \mathbf{f}(x) \lesssim_{\Omega} M_{1, \alpha} \mathbf{f}(x)$; which means $M_{\Omega, \alpha} f_{j}(x) \lesssim_{\Omega} M_{1, \alpha} f_{j}(x)$ for any $j \in \mathbb{N}$. Thus, for $\Omega \in L^{\infty}\left(S^{n-1}\right)$, we have inequality (4) and (5) immediately from Proposition 1 or Proposition 2. Therefore, we consider a weaker condition than $\Omega \in L^{\infty}\left(S^{n-1}\right)$.

Furthermore, we extend inequality (4) and (5) onto Morrey space in Section 3. Let us recall the definition of a Morrey spaces. For $p \in[1, \infty)$ and $\lambda \in[0, n)$, the Morrey norm is defined as

$$
\|f\|_{L^{p, \lambda}}:=\sup _{B(x, r) \subset \mathbb{R}^{n}} r^{-\lambda / p}\|f\|_{L^{p} B(x, r)} .
$$

The collection of any measurable function $f$ such that $\|f\|_{L^{p, \lambda}}<\infty$ is called the Morrey space $L^{p, \lambda}[9]$. In case of $\lambda=0$, the Morrey space $L^{p, 0}$ is equivalent, by norm value, to the Lebesgue space $L^{p}$. The weak Morrey space $W L^{p, \lambda}$ is the set of measurable function $f$ such that

$$
\|f\|_{W L^{p, \lambda}}:=\sup _{B(x, r)} r^{-\frac{\lambda}{p}}\|f\|_{W L^{p}(B(x, r))}<\infty .
$$

Let $|\mathbf{f}|=\left\{\left|f_{j}\right|\right\}_{j=1}^{\infty}$, and $\int_{\mathbb{R}^{n}} \mathbf{f}(x) d x=\left\{\int_{\mathbb{R}^{n}} f_{j}(x) d x\right\}_{j=1}^{\infty}$. For $u \in[1, \infty]$, one can observe that for any function $\mathbf{f} \in L^{1}\left(\ell^{u}\right)$, we have obvious inequality

$$
\|\| \mathbf{f}\left\|_{L^{1}}\right\|_{\ell^{u}} \leq\|\mathbf{f}\|_{L^{1}\left(\ell^{u}\right)}
$$


2. Extension on Lebesgue spaces. Let us begin this section with the definition of a rough integral operator $T_{\Omega, \alpha}$. Let $\alpha \in[0, n), \Omega$ be a homogeneous function of degree zero. The rough integral operator $T_{\Omega, \alpha}$ is defined as

$$
T_{\Omega, \alpha} f(x)=\int_{\mathbb{R}^{n}} \frac{\Omega(x-y)}{|x-y|^{n-\alpha}} f(y) d y .
$$

For $\alpha \in(0, n)$, the operator $T_{1, \alpha}$ is known as the fractional integral operator. The operator $T_{\Omega, 0}$ is known as the singular integral operator. A boundedness property is known.

Proposition 3 ([10], Theorem 2). Let $\alpha \in(0, n), p \in\left(1, \frac{n}{\alpha}\right)$, and $\frac{1}{q}=\frac{1}{p}-\frac{\alpha}{n}$. The operator $T_{\Omega, \alpha}$ is bounded from $L^{p}$ to $L^{q}$ if $\Omega \in L^{s}\left(S^{n-1}\right)$ where $s \geq \frac{n}{n-\alpha}$.

Let $T_{\Omega, \alpha} \mathbf{f}$ be a function sequence $\left\{T_{\Omega, \alpha} f_{j}\right\}_{j=1}^{\infty}$. It is clear that $M_{\Omega, \alpha} \mathbf{f}(x) \leq T_{|\Omega|, \alpha}(|\mathbf{f}|)(x)$. Under the same argument to obtain inequality (3), we have

$$
\left\|M_{\Omega, \alpha} \mathbf{f}(x)\right\|_{\ell^{\infty}} \leq M_{\Omega, \alpha}\left(\|\mathbf{f}(\cdot)\|_{\ell^{\infty}}\right)(x) .
$$

Hence, by inequality (6) and (7), for any $u \in[1, \infty]$

$$
\left\|M_{\Omega, \alpha} \mathbf{f}(x)\right\|_{\ell^{u}} \leq T_{|\Omega|, \alpha}\left(\|\mathbf{f}(\cdot)\|_{\ell^{u}}\right)(x) .
$$

With the relation between $M_{\Omega, \alpha}$ and $T_{\Omega, \alpha}$, we can work on inequality (4) and have the following result.

Theorem 1. Let $\alpha \in[0, n), p \in\left(1, \frac{n}{\alpha}\right), \frac{1}{q}=\frac{1}{p}-\frac{\alpha}{n}$, and $\Omega \in L^{s}\left(S^{n-1}\right)$ with $s \geq \frac{n}{n-\alpha}$. Then, for $\alpha \in(0, n)$ and $u \in[1, \infty]$, inequality (4) holds. Moreover, for $\alpha=0$ and $u \in(1, \infty]$, inequality (4) holds.

Proof. By inequality (8) and Proposition 3, we can verify the case of $\alpha \in(0, \infty)$ as follows.

$$
\left\|M_{\Omega, \alpha} \mathbf{f}\right\|_{L^{q}\left(\ell^{u}\right)} \leq\left\|T_{|\Omega|, \alpha}\left(\|\mathbf{f}(\cdot)\|_{\ell^{u}}\right)\right\|_{L^{q}} \lesssim \Omega\|\mathbf{f}\|_{L^{p}\left(\ell^{u}\right)} .
$$

We now need to clarify the case of $\alpha=0$. For each $j \in \mathbb{N}$, we have

$$
M_{\Omega, 0} f_{j}(x) \leq \int_{S^{n-1}}|\Omega(\theta)| M_{\theta} f_{j}(x) d \sigma(\theta)
$$

where

$$
M_{\theta} f_{j}(x)=\sup _{r>0} r^{-1} \int_{0}^{r}\left|f_{j}(x-R \theta)\right| d R .
$$

By the rotation method and Proposition 1 we obtain

$$
\left\|M_{\theta} \mathbf{f}\right\|_{L^{p}\left(\ell^{u}\right)} \lesssim\|\mathbf{f}\|_{L^{p}\left(\ell^{u}\right)}
$$

Using inequalities (9) and (6), we get

$$
\left\|M_{\Omega, 0} \mathbf{f}(x)\right\|_{\ell^{u}} \leq\left(\int_{S^{n-1}}|\Omega(\theta)|\left\|M_{\theta} \mathbf{f}(x)\right\|_{\ell^{u}} d \sigma(\theta)\right) .
$$


By the Minkowski inequality, and inequality (10), we proceed as follows

$$
\begin{gathered}
\left\|M_{\Omega, 0} \mathbf{f}\right\|_{L^{p}\left(\ell^{u}\right)} \leq\left\|\left(\int_{S^{n-1}}|\Omega(\theta)|\left\|M_{\theta} \mathbf{f}(\cdot)\right\|_{\ell^{u}} d \sigma(\theta)\right)\right\|_{L^{p}} \\
\leq\left(\int_{S^{n-1}}|\Omega(\theta)|\left\|M_{\theta} \mathbf{f}\right\|_{L^{p}\left(\ell^{u}\right)} d \sigma(\theta)\right) \lesssim_{\Omega}\|\mathbf{f}\|_{L^{p}\left(\ell^{u}\right)} .
\end{gathered}
$$

This completes the proof of Theorem 1 .

We can also work on inequality (5) for $\alpha \in(0, n)$ with the relation between $M_{\Omega, \alpha}$ and $T_{\Omega, \alpha}$ as follows.

Theorem 2. Let $\alpha \in(0, n)$, and $u \in[1, \infty]$. If $\Omega \in L^{s}\left(S^{n-1}\right)$ with $s \geq \frac{n}{n-\alpha}$, then inequality (5) holds.

Proof. We can rewrite the function $T_{\Omega, \alpha} f$ as $K_{\Omega, \alpha} * f$ where $K_{\Omega, \alpha}(x)=\Omega(x)|x|^{\alpha-n}$. Since $\Omega \in L^{\frac{n}{n-\alpha}}\left(S^{n-1}\right)$, we have $K_{\Omega, \alpha} \in W L^{\frac{n}{n-\alpha}}$ (see [1, p. 224]). By Young's inequality, the operator $T_{\Omega, \alpha}$ is bounded from $L^{1}$ to $W L^{\frac{n}{n-\alpha}}$ due to

$$
\left\|T_{\Omega, \alpha} f\right\|_{W L^{\frac{n}{n-\alpha}}} \leq\left\|K_{\Omega, \alpha}\right\|_{W L^{\frac{n}{n-\alpha}}}\|f\|_{L^{1}} .
$$

Therefore, by inequality (8),

$$
\left\|M_{\Omega, \alpha} \mathbf{f}\right\|_{W L^{\frac{n}{n-\alpha}\left(\ell^{u}\right)}} \leq\left\|T_{|\Omega|, \alpha}\left(\|\mathbf{f}(\cdot)\|_{\ell^{u}}\right)\right\|_{W L^{\frac{n}{n-\alpha}} \lesssim \Omega}\|\mathbf{f}\|_{L^{1}\left(\ell^{u}\right)} .
$$

The function $\Omega$ is said to satisfy $L^{1}$-Dini condition if $\Omega \in L^{1}\left(S^{n-1}\right)$ and

$$
\int_{0}^{1} \frac{w(\delta)}{\delta} d \delta<\infty
$$

where

$$
w(\delta)=\sup _{h \in \mathbb{R}^{n},|h| \leq \delta} \int_{S^{n-1}}|\Omega(\theta+h)-\Omega(\theta)| d \sigma(\theta) .
$$

Proposition 4 ([3], Theorem 1.5). $M_{\Omega, 0}$ is bounded from $L^{1}$ to $W L^{1}$ if $\Omega$ satisfies $L^{1}$-Dini condition.

We borrow some notations from [3, proof of Theorem 1.5]. Let $\phi$ be a nonnegative, radial, radially decreasing $C^{\infty}$-function such that $\phi(x)=1$ for $|x| \leq 1$, and $\phi$ supported in $\left\{x \in \mathbb{R}^{n} ;|x|<\frac{3}{2}\right\}$. Let also $\phi_{r}(x)=r^{-n} \phi\left(\frac{x}{r}\right)$. For $\Omega \geq 0$ and $f \geq 0$, we have $M_{\Omega, 0} f(x) \leq$ $M_{\Omega, 0, \phi} f(x)$ where

$$
M_{\Omega, 0, \phi} f(x)=\sup _{r>0}\left|\int_{\mathbb{R}^{n}} \phi_{r}(x-y) \Omega(x-y) f(y) d y\right| .
$$

Suppose that $A^{c}:=\mathbb{R}^{n} \backslash A$ for any $A \subset \mathbb{R}^{n}$. From [3, proof of Theorem 1.5], we can conclude the following corollary. 
Corollary 1. Suppose $f$ is supported in a cube $Q$ with center $y_{0}$ such that $\int f=0$. Then,

$$
\begin{gathered}
M_{\Omega, 0, \phi} f(x) \lesssim \int_{|x-y| \leq 2\left|y-y_{0}\right|}\left(\frac{|\Omega(x-y)|}{|x-y|^{n}}+\frac{\left|\Omega\left(x-y_{0}\right)\right|}{\left|x-y_{0}\right|^{n}}\right)|f(y)| d y+ \\
+\int_{|x-y|>2\left|y-y_{0}\right|} \frac{\left|\Omega(x-y)-\Omega\left(x-y_{0}\right)\right|}{|x-y|^{n}}|f(y)| d y+ \\
+\int_{|x-y|>2\left|y-y_{0}\right|} \frac{\left|y-y_{0}\right|}{\left|x-y_{0}\right|^{n+1}}\left|\Omega\left(x-y_{0}\right)\right||f(y)| d y=F_{1}(f)(x)+F_{2}(f)(x)+F_{3}(f)(x) .
\end{gathered}
$$

Let $\tilde{Q}$ with the same center as cube $Q$, such that the sides of $\tilde{Q}$ are parallel to the sides of $Q$, and the side-length $\ell(\tilde{Q})=2 \ell(Q)$. If $\Omega$ satisfies $L^{1}$-Dini condition, then for each $i \in\{1,2,3\}$

$$
\int_{\tilde{Q}^{c}}\left|F_{i}(f)(x)\right| d x \lesssim \Omega\|f\|_{L^{1}}
$$

We now can prove inequality (5) for $\alpha=0$.

Theorem 3. For $\alpha=0$, and $u \in(1, \infty]$, if $\Omega$ satisfies $L^{1}$-Dini condition, then inequality (5) holds.

Proof. The case of $u=\infty$ is clear by Proposition 4 and inequality (7).

$$
\left\|M_{\Omega, 0} \mathbf{f}\right\|_{W L^{1}\left(\ell^{\infty}\right)} \leq\left\|M_{\Omega, 0}\left(\|\mathbf{f}(\cdot)\|_{\ell^{\infty}}\right)\right\|_{W L^{1}} \lesssim \Omega\|\mathbf{f}\|_{L^{1}\left(\ell^{\infty}\right)} .
$$

What is left is the case of $u \in(1, \infty)$.

Without loss of generality, we may suppose $\Omega \geq 0$ and $\mathbf{f} \geq \mathbf{0}$ where $\mathbf{0}$ is the zero function sequence. Fix $\lambda>0$. Since $\|\mathbf{f}(\cdot)\|_{\ell^{u}} \in L^{1}$, by applying the covering property (see [4, Theorem 2.11]), we have a collection of dyadic cubes $\left\{Q_{k}\right\}$ (may be empty) such that

(i) $\|\mathbf{f}(x)\|_{\ell^{u}} \leq \lambda$ for almost every $x \notin \Psi=\bigcup_{k} Q_{k}$.

(ii) $|\Psi|<\frac{1}{\lambda}\|\mathbf{f}\|_{L^{1}\left(\ell^{u}\right)}$.

(iii) $\lambda<\frac{1}{\left|Q_{k}\right|} \int_{Q_{k}}\|\mathbf{f}(y)\|_{\ell^{u}} d y \leq 2^{n} \lambda$.

We decompose $\mathbf{f}=\left\{f_{j}\right\}=\left\{g_{j}\right\}+\left\{b_{j}\right\}=\mathbf{g}+\mathbf{b}$ where

$$
g_{j}=f_{j} \chi_{\Psi^{c}}+\sum_{k} \frac{\chi_{Q_{k}}}{\left|Q_{k}\right|} \int_{Q_{k}}\left|f_{j}(y)\right| d y
$$

and $b_{j}=\sum_{k} b_{j, k}$ with

$$
b_{j, k}=f_{j} \chi_{Q_{k}}-\frac{\chi_{Q_{k}}}{\left|Q_{k}\right|} \int_{Q_{k}}\left|f_{j}(y)\right| d y .
$$

We write $\mathbf{b}=\left\{b_{j}\right\}=\sum_{k}\left\{b_{j, k}\right\}=\sum_{k} \mathbf{b}_{k}$. Let us take a look on behavior of $\mathbf{g}$ and $\mathbf{b}$. By inequality (6), (i) and (iii)

$$
\|\mathbf{g}\|_{L^{u}\left(\ell^{u}\right)}^{u}=\int_{\Psi^{c}}\|\mathbf{g}(x)\|_{\ell^{u}}^{u} d x+\sum_{k} \int_{Q_{k}}\|\mathbf{g}(x)\|_{\ell^{u}}^{u} d x \leq
$$




$$
\leq \int_{\Psi^{c}}\|\mathbf{f}(x)\|_{\ell^{u}}^{u} d x+\sum_{k} \int_{Q_{k}} \frac{1}{\left|Q_{k}\right|^{u}}\left(\int_{Q_{k}}\|\mathbf{f}(y)\|_{\ell^{u}} d y\right)^{u} d x \lesssim \lambda^{u-1}\|\mathbf{f}\|_{L^{1}\left(\ell^{u}\right)} .
$$

For any $k$, we have $\int b_{j, k}=0$. Moreover, by inequality (6)

$$
\sum_{k}\left\|\mathbf{b}_{k}\right\|_{L^{1}\left(\ell^{u}\right)} \lesssim\|\mathbf{f}\|_{L^{1}\left(\ell^{u}\right)}
$$

Since $M_{\Omega, 0} f(x) \leq M_{\Omega, 0, \phi} f(x)$ (see (12)), we now only need to show

$$
\left|\left\{x \in \mathbb{R}^{n} ;\left\|M_{\Omega, 0, \phi} \mathbf{g}(x)\right\|_{\ell^{u}}>\frac{\lambda}{2}\right\}\right| \lesssim \Omega \frac{1}{\lambda}\|\mathbf{f}\|_{L^{1}\left(\ell^{u}\right)}
$$

and

$$
\left|\left\{x \in \mathbb{R}^{n} ;\left\|M_{\Omega, 0, \phi} \mathbf{b}(x)\right\|_{\ell^{u}}>\frac{\lambda}{2}\right\}\right| \lesssim \Omega \frac{1}{\lambda}\|\mathbf{f}\|_{L^{1}\left(\ell^{u}\right)} .
$$

First, let us prove inequality (15). By Chebychev's inequality, inequality (6), Minkowski's inequality, inequalities (10) and (13), we have

$$
\begin{gathered}
\left|\left\{x \in \mathbb{R}^{n} ;\left\|M_{\Omega, 0, \phi} \mathbf{g}(x)\right\|_{\ell^{u}}>\frac{\lambda}{2}\right\}\right| \lesssim \frac{1}{\lambda^{u}}\left\|M_{\Omega, 0, \phi} \mathbf{g}\right\|_{L^{u}\left(\ell^{u}\right)}^{u} \lesssim \\
\lesssim \frac{1}{\lambda^{u}}\left(\int_{S^{n-1}}|\Omega(\theta)|\left\|M_{\theta} \mathbf{g}\right\|_{L^{u}\left(\ell^{u}\right)} d \sigma(\theta)\right)^{u} \lesssim \frac{1}{\lambda^{u}}\|\mathbf{g}\|_{L^{u}\left(\ell^{u}\right)}^{u} \lesssim \frac{1}{\lambda}\|\mathbf{f}\|_{L^{1}\left(\ell^{u}\right)} .
\end{gathered}
$$

Recall $\tilde{Q}$ from Corollary 1. Suppose that $\tilde{\Psi}=\bigcup_{k} \tilde{Q}_{k}$. From (ii), we have

$$
|\tilde{\Psi}| \lesssim|\Psi| \leq \frac{1}{\lambda}\|\mathbf{f}\|_{L^{1}\left(\ell^{u}\right)}
$$

Since we have inequality (17), we can get inequality (16) once we show the following.

$$
\left|\left\{x \in \tilde{\Psi}^{c} ;\left\|M_{\Omega, 0, \phi} \mathbf{b}(x)\right\|_{\ell^{u}}>\frac{\lambda}{2}\right\}\right| \lesssim \Omega \frac{1}{\lambda}\|\mathbf{f}\|_{L^{1}\left(\ell^{u}\right)} .
$$

By Chebychev's inequality and Minkowski's inequality

$$
\begin{gathered}
\left|\left\{x \in \tilde{\Psi}^{c} ;\left\|M_{\Omega, 0, \phi} \mathbf{b}(x)\right\|_{\ell^{u}}>\frac{\lambda}{2}\right\}\right| \lesssim \frac{1}{\lambda} \int_{\tilde{\Psi}^{c}}\left\|M_{\Omega, 0, \phi} \mathbf{b}(x)\right\|_{\ell^{u}} d x \leq \\
\leq \frac{1}{\lambda} \sum_{k} \int_{\tilde{\Psi}^{c}}\left\|M_{\Omega, 0, \phi} \mathbf{b}_{k}(x)\right\|_{\ell^{u}} d x .
\end{gathered}
$$

Let us consider a fixed $b_{j, k}$ which is supported in $Q_{k}$. Suppose that $y_{0}$ is the center of $Q_{k}$. Since the integral of $b_{j, k}$ is zero, by Corollary 1

$$
M_{\Omega, 0, \phi} b_{j, k}(x) \lesssim F_{1}\left(b_{j, k}\right)(x)+F_{2}\left(b_{j, k}\right)(x)+F_{3}\left(b_{j, k}\right)(x) .
$$


By inequality (6), and the boundedness of $F_{i}$ for each $i \in\{1,2,3\}$ from Corollary 1 , we can conclude that

$$
\int_{\tilde{\Psi}^{c}}\left(\sum_{j=1}^{\infty}\left|F_{i}\left(b_{j, k}\right)(x)\right|^{u}\right)^{\frac{1}{u}} d x \leq \int_{{\tilde{Q_{k}}}^{c}} F_{i}\left(\left\|\mathbf{b}_{k}(\cdot)\right\|_{\ell^{u}}\right)(x) d x \lesssim \Omega\left\|\mathbf{b}_{k}\right\|_{L^{1}\left(\ell^{u}\right)} .
$$

Hence, from inequality (18), and (14),

$$
\left|\left\{x \in \tilde{\Psi}^{c} ;\left\|M_{\Omega, 0, \phi} \mathbf{b}(x)\right\|_{\ell^{u}}>\frac{\lambda}{2}\right\}\right| \lesssim \Omega \frac{1}{\lambda} \sum_{k}\left\|\mathbf{b}_{k}\right\|_{L^{1}\left(\ell^{u}\right)} \lesssim \frac{1}{\lambda}\|\mathbf{f}\|_{L^{1}\left(\ell^{u}\right)} .
$$

Thus, inequality (16) is verified. This completes the proof of Theorem 3.

3. Extension on Morrey spaces. Let us start this section with some properties of the rough integral operator $T_{\Omega, \alpha}$. Let $p^{\prime}$ be the exponent conjugate to $p$ where $p^{\prime}=\frac{p}{p-1}$.

Proposition 5 ([11], Lemma 3.2). Let $\alpha \in[0, n), \lambda, \mu \in[0, n), p \in\left(1, \frac{n-\lambda}{\alpha}\right)$, and $\frac{n-\mu}{q}=$ $\frac{n-\lambda}{p}-\alpha$. Let also $\Omega \in L^{s}\left(S^{n-1}\right)$ with $s \geq p^{\prime}$. Then for any $z \in \mathbb{R}^{n}$ and $r>0$

$$
r^{-\frac{\mu}{q}}\left\|T_{\Omega, \alpha}\left(f \chi_{B^{c}(z, 2 r)}\right)\right\|_{L^{q}(B(z, r))} \lesssim_{\Omega}\|f\|_{L^{p, \lambda}}
$$

Lemma 1. Let $\alpha \in[0, n), s \in(1, \infty), p \in[1, \infty), \lambda \in\left[0, n-\frac{n p}{s}-\alpha p\right), \mu \in[0, n)$, and $\frac{n-\mu}{q}=\frac{n-\lambda}{p}-\alpha$. Let also $\Omega \in L^{s}\left(S^{n-1}\right)$. Then for any $z \in \mathbb{R}^{n}$ and $r>0$

$$
r^{-\frac{\mu}{q}}\left\|T_{\Omega, \alpha}\left(f \chi_{B^{c}(z, 2 r)}\right)\right\|_{L^{q}(B(z, r))} \lesssim \Omega\|f\|_{L^{p, \lambda}}
$$

Proof. For any $x \in B(z, r)$ we have $B(x, t) \subset B(z, 2 t)$ for $t>r$. By Fubini's theorem,

$$
\begin{gathered}
\left|T_{\Omega, \alpha}\left(f \chi_{B^{c}(z, 2 r)}\right)(x)\right| \leq \int_{B^{c}(z, 2 r)} \frac{|\Omega(x-y)|}{|x-y|^{n-\alpha}}|f(y)| d y \lesssim \\
\lesssim \int_{B^{c}(z, 2 r)}|\Omega(x-y)||f(y)| \int_{|x-y|}^{\infty} t^{\alpha-n-1} d t d y \leq \int_{r}^{\infty} t^{\alpha-n-1} \int_{B(x, t)}|\Omega(x-y)||f(y)| d y d t \leq \\
\leq \int_{r}^{\infty} t^{\alpha-n-1} \int_{B(z, 2 t)}|\Omega(x-y)||f(y)| d y d t .
\end{gathered}
$$

Since $y \in B(z, 2 t), B(z, r) \subset B(y, 4 t)$ for $t>r$. Since $\lambda<n-\frac{n p}{s}-\alpha p$ and $\frac{n-\mu}{q}=\frac{n-\lambda}{p}-\alpha$, we have $s>q$. Applying inequality (19), Minkowski and Hölder inequalities, we have

$$
\begin{gathered}
r^{-\frac{\mu}{q}}\left\|T_{\Omega, \alpha}\left(f \chi_{B^{c}(z, 2 r)}\right)\right\|_{L^{q}(B(z, r))} \\
\leq r^{-\frac{\mu}{q}} \int_{r}^{\infty} t^{\alpha-n-1} \int_{B(z, 2 t)}|f(y)|\left(\int_{B(z, r)}|\Omega(x-y)|^{q} d x\right)^{\frac{1}{q}} d y d t \lesssim
\end{gathered}
$$




$$
\begin{aligned}
& \lesssim r^{\frac{n-\mu}{q}-\frac{n}{s}} \int_{r}^{\infty} t^{\alpha-n-1} \int_{B(z, 2 t)}|f(y)|\left(\int_{B(z, r)}|\Omega(x-y)|^{s} d x\right)^{\frac{1}{s}} d y d t \leq \\
& \leq r^{\frac{n-\mu}{q}-\frac{n}{s}} \int_{r}^{\infty} t^{\alpha-n-1} \int_{B(z, 2 t)}^{\infty}|f(y)|\left(\int_{B(y, 4 t)}|\Omega(x-y)|^{s} d x\right)^{\frac{1}{s}} d y d t \lesssim \Omega \\
& \lesssim r^{\frac{n-\mu}{q}-\frac{n}{s}} \int_{r}^{\infty} t^{\alpha-n+\frac{n}{s}-1} \int_{B(z, 2 t)}|f(y)| d y d t \lesssim \Omega \\
& \lesssim r^{\frac{n-\mu}{q}-\frac{n}{s}} \int_{r}^{\infty} t^{\alpha-\frac{n}{p}+\frac{n}{s}-1}\|f\|_{L^{p}(B(z, 2 t))} d t \leq\|f\|_{L^{p, \lambda}} r^{\frac{n-\mu}{q}-\frac{n}{s}} \int_{r}^{\infty} t^{\alpha-\frac{n-\lambda}{p}+\frac{n}{s}-1} d t .
\end{aligned}
$$

Since $\lambda<n-\frac{n p}{s}-\alpha p$, we get the desired result.

We note that the proof of Lemma 1 is similar to Guliyev's result in [7, p. 217]. Guliyev used $\|\Omega(\cdot-y)\|_{L^{s}(B(z, r))} \sim r^{\frac{n}{s}}\|\Omega\|_{L^{s}\left(S^{n-1}\right)}$. However, we can not simply do the same way since $\|\Omega(\cdot-y)\|_{L^{s}(B(z, r))}$ depends on $y$. Therefore, we instead treat inequality (20) by the fact that $B(z, r) \subset B(y, 4 t)$, and

$$
\|\Omega(\cdot-y)\|_{L^{s}(B(y, 4 t))}=\|\Omega\|_{L^{s}(B(0,4 t))} \sim t^{\frac{n}{s}}\|\Omega\|_{L^{s}\left(S^{n-1}\right)} .
$$

We have two following boundedness properties of $T_{\Omega, \alpha}$.

Proposition 6 ([11], Theorem 3.3). Let $\alpha \in(0, n), 0 \leq \lambda \leq \mu<n, p \in\left(1, \frac{n-\lambda}{\alpha}\right)$, and $\frac{n-\mu}{q}=\frac{n-\lambda}{p}-\alpha$. Let $\Omega \in L^{s}\left(S^{n-1}\right)$ where $s \geq p^{\prime}$. Then $T_{\Omega, \alpha}$ is bounded from $L^{p, \lambda}$ to $L^{q, \mu}$.

Theorem 4. Let $\alpha \in(0, n), p, s \in(1, \infty), \lambda \in\left[0, n-\frac{n p}{s}-\alpha p\right), \mu \in\left[\frac{\lambda q}{p}, n\right)$, and $\frac{n-\mu}{q}=\frac{n-\lambda}{p}-\alpha$. Let also $\Omega \in L^{s}\left(S^{n-1}\right)$. Then $T_{\Omega, \alpha}$ is bounded from $L^{p, \lambda}$ to $L^{q, \mu}$.

Proof. Since $\mu \geq \frac{\lambda q}{p}$, we have $q_{1} \geq q$ with $\frac{1}{q_{1}}=\frac{1}{p}-\frac{\alpha}{n}$. Fix $z \in \mathbb{R}^{n}$ and $r>0$. We define $f_{1}=f \chi_{B(z, 2 r)}$ and $f_{2}=f-f_{1}$. By the Hölder inequality and Proposition 3, we have

$$
\begin{gathered}
r^{-\frac{\mu}{q}}\left\|T_{\Omega, \alpha} f_{1}\right\|_{L^{q}(B(z, r))} \lesssim r^{\frac{n-\mu}{q}-\frac{n}{q_{1}}}\left\|T_{\Omega, \alpha} f_{1}\right\|_{L^{q_{1}}(B(z, r))} \lesssim \Omega \\
\lesssim \Omega r^{\frac{n-\mu}{q}-\frac{n}{v}}\left\|f_{1}\right\|_{L^{p}(B(z, 2 r))} \lesssim\|f\|_{L^{p, \lambda}}
\end{gathered}
$$

Due to inequality (21) and Lemma 1, we have

$$
r^{-\frac{\mu}{q}}\left\|T_{\Omega, \alpha} f\right\|_{L^{q}(B(z, r))} \leq r^{-\frac{\mu}{q}}\left(\left\|T_{\Omega, \alpha} f_{1}\right\|_{L^{q}(B(z, r))}+\left\|T_{\Omega, \alpha} f_{2}\right\|_{L^{q}(B(z, r))}\right) \lesssim \Omega\|f\|_{L^{p, \lambda}} .
$$

By the properties of $T_{\Omega, \alpha}$, we can extend inequality (4) onto Morrey spaces as follows.

Theorem 5. Let $\alpha \in[0, n), 0 \leq \lambda \leq \mu<n, p \in\left(1, \frac{n-\lambda}{\alpha}\right), \frac{n-\mu}{q}=\frac{n-\lambda}{p}-\alpha$, and $\Omega \in L^{s}\left(S^{n-1}\right)$ with $s \geq p^{\prime}$. Then, for $\alpha \in(0, n)$ and $u \in[1, \infty]$,

$$
\left\|M_{\Omega, \alpha} \mathbf{f}\right\|_{L^{q, \mu}\left(\ell^{u}\right)}:=\|\| M_{\Omega, \alpha} \mathbf{f}(\cdot)\left\|_{\ell^{u}}\right\|_{L^{q, \mu}} \lesssim \Omega\|\mathbf{f}\|_{L^{p, \lambda}\left(\ell^{u}\right)} .
$$

Moreover, for $\alpha=0$ and $u \in(1, \infty]$, inequality (22) holds. 
Theorem 6. Let $\alpha \in[0, n), p \in(1, \infty), s \in(1, \infty), \lambda \in\left[0, n-\frac{n p}{s}-\alpha p\right), \mu \in\left[\frac{\lambda q}{p}, n\right)$, $\frac{n-\mu}{q}=\frac{n-\lambda}{p}-\alpha$, and $\Omega \in L^{s}\left(S^{n-1}\right)$. Then, for $\alpha \in(0, n)$ and $u \in[1, \infty]$, inequality (22) holds. For $\alpha=0$ and $u \in(1, \infty]$, inequality (22) holds.

Proof of Theorem 5 and Theorem 6 . The case of $\alpha \in(0, n)$ is verified by inequality (8), Proposition 6 (for Theorem 5), and Theorem 4 (for Theorem 6).

$$
\left\|M_{\Omega, \alpha} \mathbf{f}\right\|_{L^{q, \mu}\left(\ell^{u}\right)} \leq\left\|T_{|\Omega|, \alpha}\left(\|\mathbf{f}(\cdot)\|_{\ell^{u}}\right)\right\|_{L^{q, \mu}} \lesssim \Omega\|\mathbf{f}\|_{L^{p, \lambda}\left(\ell^{u}\right)} .
$$

Let us now treat the case of $\alpha=0$. Fix $B(z, r) \subset \mathbb{R}^{n}$. Suppose that $\mathbf{f}=\left\{f_{j}\right\}=\left\{f_{j,(0)}+\right.$ $\left.f_{j,(1)}\right\}=\mathbf{f}_{(0)}+\mathbf{f}_{(1)}$, where $f_{j,(0)}=f_{j} \chi_{B(z, 2 r)}$ and $f_{j,(1)}=f_{j}-f_{j,(0)}$. Since $M_{\Omega, 0}$ is a sublinear operator, we have

$$
\left\|M_{\Omega, 0} \mathbf{f}(x)\right\|_{\ell^{u}} \leq\left\|M_{\Omega, 0} \mathbf{f}_{(0)}(x)\right\|_{\ell^{u}}+\left\|M_{\Omega, 0} \mathbf{f}_{(1)}(x)\right\|_{\ell^{u}} .
$$

For the first term on the right-hand side of inequality (23), we use the Hölder inequality with order $p / q$ and Theorem 1 to obtain

$$
r^{-\frac{\mu}{q}}\|\| M_{\Omega, 0} \mathbf{f}_{(0)}(\cdot)\left\|_{\ell^{u}}\right\|_{L^{q}(B(z, r))} \lesssim r^{-\frac{n}{p}+\frac{n-\mu}{q}}\|\| M_{\Omega, 0} \mathbf{f}_{(0)}(\cdot)\left\|_{\ell^{u}}\right\|_{L^{p}(B(z, r))} \lesssim \Omega\|\mathbf{f}\|_{L^{p, \lambda}\left(\ell^{u}\right)} .
$$

Now, we treat the second term. By virtue of inequality (8), Proposition 5 (for Theorem 5), and Lemma 1 (for Theorem 6), we have

$$
r^{-\frac{\mu}{q}}\|\| M_{\Omega, 0} \mathbf{f}_{(1)}(\cdot)\left\|_{\ell^{u}}\right\|_{L^{q}(B(z, r))} \leq r^{-\frac{\mu}{q}}\left\|T_{|\Omega|, \alpha}\left(\left\|\mathbf{f}_{(1)}(\cdot)\right\|_{\ell^{u}}\right)\right\|_{L^{q}(B(z, r))} \lesssim \Omega\|\mathbf{f}\|_{L^{p, \lambda}\left(\ell^{u}\right)} .
$$

This completes the proof of both Theorem 5 and Theorem 6 .

We also can extend inequality (5) onto weak Morrey spaces as follows.

Theorem 7. Let $\alpha \in[0, n), q=\frac{n-\mu}{n-\lambda-\alpha}<s, \lambda \in\left[0, n-\frac{n}{s}-\alpha\right)$, and $\mu \in[\lambda q, n)$. For $\alpha \in(0, n)$ and $u \in[1, \infty]$, if $\Omega \in L^{s}\left(S^{n-1}\right)$, then

$$
\left\|M_{\Omega, \alpha} \mathbf{f}\right\|_{W L^{q, \mu}\left(\ell^{u}\right)}:=\|\| M_{\Omega, \alpha} \mathbf{f}(\cdot)\left\|_{\ell^{u}}\right\|_{W L^{q, \mu}} \lesssim \Omega\|\mathbf{f}\|_{L^{1, \lambda}\left(\ell^{u}\right)} .
$$

For $\alpha=0$ and $u \in(1, \infty]$, if $\Omega \in L^{s}\left(S^{n-1}\right)$ satisfies $L^{1}$-Dini condition, then inequality (24) holds.

Proof. Since $\mu>\lambda q$, we have $q_{1}=\frac{n}{n-\alpha}>q$. We use the same decomposition as in inequality (25). For the first term of right-hand side in inequality (23), we use the Hölder inequality with the exponent $q_{1} / q$, Theorem 2 (for $\alpha \in(0, n)$ ) and Theorem 3 (for $\alpha=0$ ) to obtain

$$
r^{-\frac{\mu}{q}}\|\| M_{\Omega, \alpha} \mathbf{f}_{(0)}(\cdot)\left\|_{\ell^{u}}\right\|_{W L^{q}(B(z, r))} \lesssim r^{\frac{n-\mu}{q}-\frac{n}{q_{1}}}\|\| M_{\Omega, \alpha} \mathbf{f}_{(0)}(\cdot)\left\|_{\ell^{u}}\right\|_{W L^{q_{1}(B(z, r))}} \lesssim \Omega\|\mathbf{f}\|_{L^{1, \lambda}\left(\ell^{u}\right)} .
$$

Let us now treat the second term. For any $\gamma>0$, by Chebychev's inequality, inequality (8), and Lemma 1, we have

$$
\begin{gathered}
r^{-\frac{\mu}{q}}\left|\left\{x \in B(z, r) ;\left\|M_{\Omega, \alpha} \mathbf{f}_{(1)}(x)\right\|_{\ell^{u}}>\gamma\right\}\right|^{\frac{1}{q}} \leq \\
\leq \frac{r^{-\frac{\mu}{q}}}{\gamma}\left\|T_{|\Omega|, \alpha}\left(\left\|\mathbf{f}_{(1)}(\cdot)\right\|_{\ell^{u}}\right)\right\|_{L^{q}(B(z, r))} \lesssim \Omega \frac{1}{\gamma}\|\mathbf{f}\|_{L^{1, \lambda}\left(\ell^{u}\right)} .
\end{gathered}
$$

This completes the proof of Theorem 7 . 
4. Remarks. To the best of our knowledge, there is still no proof for $\Omega \in L^{1}\left(S^{n-1}\right)$ to be sufficient condition for boundedness of $M_{\Omega, 0}$ from $L^{1}$ to $W L^{1}$. Moreover, there is still no counterexample such that $M_{\Omega, 0}: L^{1} \rightarrow W L^{1}$ is not bounded under $\Omega \in L^{1}\left(S^{n-1}\right)$ either. Therefore, possibility of removing Dini-condition from Theorem 3 and Theorem 6 is still an open problem. Furthermore, the necessary condition (in term of $\Omega$ ) for inequality (4), inequality (5), inequality (22), or inequality (24) to hold is still open .

Acknowledgment. This research was supported by P3MI ITB 2018 Grant.

\section{REFERENCES}

1. Chanillo S., Watson D., Wheeden R., Some integral and maximal operators related to starlike sets, Studia Math., 3 (1993), №107, 223-255.

2. Chiarenza F., Frasca M., Morrey spaces and Hardy-Littlewood maximal function, Rend. Mat., 7 (1987), 273-279.

3. Ding Y., Lai X., Weak type (1,1) behavior for the maximal operator with $L^{1}$-Dini kernel, Potential Anal., 47 (2017), №2, 169-187.

4. Duoandikoetxea J., Fourier analysis. American Mathematical Soc V.29, 2001.

5. Fefferman C., Stein E.M., Some maximal inequalities, Am. J. Math., 93 (1971), №1, 107-115.

6. Grafakos L., Classical fourier analysis, V.2, Springer, New York, 2008.

7. Guliyev, Vagif S., Generalized local Morrey spaces and fractional integral operators with rough kernel, J. Math. Sci. (N. Y.), 193 (2013), №2, 211-227.

8. Iida T., Weighted inequalities on Morrey spaces for linear and multilinear fractional integrals with homogeneous kernels, Taiwan J. Math., 18 (2014), №1, 147-185.

9. Morrey C.B., Functions of several variables and absolute continuity, Duke Math. J., 6 (1940), №1, 187215.

10. Muckenhoupt B., Wheeden R.L., Weighted norm inequalities for singular and fractional integrals, Trans. Am. Math. Soc., 161 (1971), 249-258.

11. Salim D., Soeharyadi Y., Budhi W.S., Rough fractional integral operators and beyond Adams inequalities, Math. Inequal. Appl., 22 (2019), №2, 747-760.

12. Sawano Y., $\ell^{q}$-valued extension of the fractional maximal operators for non-doubling measures via potential operators, Int. J. Pure Appl. Math., 26 (2006), №4, 505-522.

13. Stein E.M., Harmonic Analysis: Real-variable Methods, Orthogonality, and Oscillatory Integrals. Princeton Mathematical Series, V.43, Princenton, University Press, Princeton, NJ, 1993.

Analysis and Geometry Research Division

Bandung Institute of Technology

Indonesia

daniel.salim@sci.ui.ac.id

wono@math.itb.ac.id

yudish@math.itb.ac.id 